\title{
Picosecond Solvation Dynamics of Coumarin153 in Bis(1-methyl- 1H-imidazol-3-ium-3-yl)dihydroborate Cation Containing Room Temperature Ionic Liquid and Ionic Liquid-DMF Mixtures
}

\author{
Prabhat Kumar Sahu, Sudhir Kumar Das and Moloy Sarkar*
}

\begin{abstract}
School of Chemical Sciences, National Institute of Science Education and Research, Bhubaneswar 751005, Orissa, India
\end{abstract}

\begin{abstract}
Steady state and time-resolved fluorescence behavior of coumarin153(C153) in bis(1-methyl-1H-imidazol-3ium-3-yl)dihydroborate cation containing room temperature ionic liquid and its mixture with dimethylformamide (DMF) has been investigated. Density functional calculations on the present ionic liquid have been carried out to have ground state structural information of this system. $\mathrm{C}-\mathrm{H} \cdots \mathrm{N}$ and $\mathrm{C}-\mathrm{H} \cdots \mathrm{O}$ hydrogen bonding interactions between cationic and anionic moiety of the present ionic liquid has been observed. Steady state absorption and emission spectral profiles of C153 are found not to be influenced by the polar cosolvent. Time-resolved fluorescence anisotropy experiments show that the rotational motion of the probe becomes faster in presence of DMF. During time dependent dynamic Stokes shift measurements in ionic liquid-DMF mixtures, the average solvation time is found to decrease with the addition of DMF to the ionic liquid. The decrease in both average solvation and rotational time of probe molecule upon gradual addition of polar organic co-solvent is attributed to the lowering of bulk viscosity of the medium.
\end{abstract}

Keywords: Ionic liquid, Time-dependent Stokes shift, Solvation dynamics, polar cosolvent, Anisotropy.

\section{INTRODUCTION}

In recent years, room-temperature ionic liquids (RTILs) have drawn great attention due to its versatile applications in the fields of chemical, material and biological sciences [1, 2]. Curiosity about their structure - property correlations have also motivated many researchers to carry out experimental and theoretical studies on these systems [3-13]. However, many of the studies have been carried out by taking neat room temperature ionic liquid as the medium. Interestingly, over the past few years, mixed ionic liquid-cosolvent systems are found to be extremely useful in several new applications, such as organic synthesis [14], catalysis [15], polymerization processes [16], extraction of solutes from solvent $[17,18]$ etc. Keeping in mind the uses of RTIL-cosolvent systems in new potential applications, it is of paramount importance to have thorough understanding of the roles of cosolvents that they play in governing the properties of RTILs.

Some studies have been carried out to understand the physicochemical properties of the RTILs-cosolvents mixtures [19-26]. It has been found out that viscosity [19], polarity [20-22], solvation [23, 24] and electrochemical $[25,26]$ behavior of the ionic liquid undergo perceptible changes in presence of cosolvents. Since solute reactivity is intricately related to solvation dynamics, studies on dynamics of solvation

${ }^{*}$ Address correspondence to this author at the School of Chemical Sciences, National Institute of Science Education and Research, Bhubaneswar 751005, Orissa, India; Tel: +91-674-2304037; Fax: +91-674-2304050;

E-mail: moloysarkar@gmail.com by monitoring the time-dependent fluorescence Stokes shift (TDFSS) of a dipolar solute in the given medium $[6,8-11]$ have been carried out to understand the dynamical features of IL-cosolvent mixtures. In this regard Paul et al. [6] have studied the effect of nonpolar toluene and dioxane on the solvation dynamics and solute rotation in 1-butyl-3methylimidazolium hexafluorophate. The composition dependence of Stokes shift dynamics of a fluorescent dye molecule in IL-cosolvent mixtures has been investigated theoretically by Ranjit Biswas et al. [12]. The theory demonstrates the dynamic Stokes shift as a sum of contributions from the dye-RTIL and the dyepolar cosolvent interactions. The theory also suggests significant contribution from solute-cation dipole-dipole interaction in solvation energy relaxation. Moreover, Stokes shift dynamics in ([Bmim][BF4]+ dichloromethane) binary mixtures predicts a very weak nonlinear composition dependence.

In this context, it may be mentioned that Sarkar and coworkers' [8-11] have studied the effects of polar solvents such as, acetonitrile, water and methanol on the dynamics of solvation of ammonium and imadazolim-based ionic liquids. These studies have demonstrated that addition of cosolvents in RTILs affects both the solvent relaxation as well as solute rotation. Very recently, we have also investigated the solvation and rotational relaxation behavior of a dipolar probe in ultra hydrophobic 1-(2-Methoxyethyl)-1methylpyrrolidinium tris(pentafluorethyl)trifluorophosphate and its mixture with toluene [13]. It has been observed that toluene can effectively penetrate into the 
ionic liquid-rich cybotactic region of the probe. This has been explained by considering favorable noncovalent interaction between ionic liquid and toluene. One general observation of the TDFSS studies is that the addition of a cosolvent to ionic liquid decreases the average solvation time and rotational time of the probe due to the lowering of viscosity of the media.

Dimethylformamide (DMF) is an interesting solvent with a strong electron-pair donating and accepting ability. It has been used to dissolve a wide range of organic and inorganic compounds which in turn has also made this solvent to be used in variety of industrial processes [27-29]. Moreover, it has been widely used in developing solvent reactivity relationships [30-32]. In view of this, it is expected that the RTIL-DMF will serve as an excellent solvent system for several applications. Very recently, Anil Kumar and coworkers have studied the thermophysical properties of binary mixtures of DMF with ammonium and imidazolium based ionic liquids [33]. However, to the best of our knowledge there is no report of solvent and rotational relaxation studies by taking DMF as cosolvent for RTILs. We would also like to take a note here that, RTIL-cosolvent systems are known to be micro-heterogeneous [34-37]. In this regard, it would also be an interesting study to find a relationship between average solvation and rotational time and medium viscosity.

Keeping all these in mind, we have investigated the solvation and rotational relaxation of Coumarin 153 (C153) in Bis(1-methyl-1H-imidazol-3-ium-3yl)dihydroborate bis(trifluoromethylsulfonyl)amide and RTIL-DMF mixtures. The present RTIL is an interesting one in a sense that two methylimidazolium cationic moieties are bridged by a borohydride group and the cationic charge is compensated by $\mathrm{NTf}_{2}{ }^{-}$. In all previous cases, solvation and rotational dynamics were studied using monocationic imidazolium moiety. The probe $\mathrm{C} 153$ used in the present study is a well-known solvatochromic probe suitable for dynamic Stokes shift

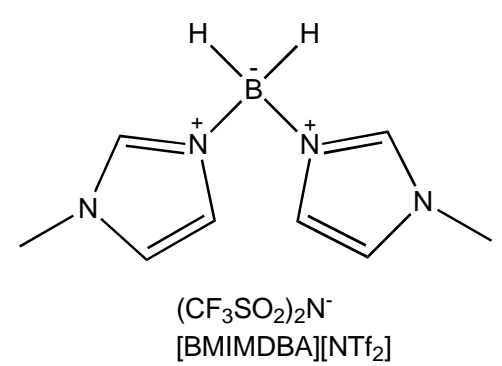

measurements [38]. The molecular diagram of RTIL and $\mathrm{C} 153$ are shown in Figure $\mathbf{1 .}$

\section{EXPERIMENTAL SECTION}

\section{Materials}

C153 (laser grade, Exciton) was used as received. The ionic liquid was obtained from Frontier Scientific Chemicals, USA (95\% purity) and purity of IL was confirmed from ${ }^{1} \mathrm{H}$ NMR spectrum. The IL was kept in high vacuum at $60^{\circ}-70^{\circ} \mathrm{C}$ for one day to remove any moisture present in this solvent. The IL was taken in a long-necked quartz cuvette and requisite amount of fluorophore was added to prepare the solution. Proper precaution was taken to avoid moisture absorption by the media during transferring the solute into the cuvette. During measurements, the concentration of the dye molecule (C153) was kept low so as to prevent dye aggregation.

\section{Instrumentation}

The viscosity of the RTIL was measured by LVDVIII Ultra Brookfield Cone and Plate viscometer (1\% accuracy and $0.2 \%$ repeatability). ${ }^{1} \mathrm{H}$ NMR spectra were obtained on a Spectrometer Bruker AVANCE 400 NMR Spectrometer at ambient temperature using tetramethylsilane (TMS) as an internal standard. ${ }^{1} \mathrm{H}$ NMR (DMSO- $\left.\mathrm{d}_{6}, 400 \mathrm{MHz}\right): \delta(\mathrm{ppm})=8.65(\mathrm{~s}, 2 \mathrm{H})$, $7.51(\mathrm{~m}, 2 \mathrm{H}), 7.35(\mathrm{~m}, 2 \mathrm{H}), 3.79(\mathrm{~s}, 6 \mathrm{H})$. The steady state absorption spectra were recorded on Perkin Elmer, Lambda-750 UV/VIS spectrophotometer and fluorescence spectra were recorded on a Perkin Elmer, LS 55 spectrofluorimeter. Time-resolved fluorescence measurements were carried out using a time-correlated single-photon counting (TCSPC) spectrometer (Edinburgh, OB920). Diode laser $\left(\lambda_{\mathrm{exc}}=405 \mathrm{~nm}\right.$, FWHM = 98 ps) was used to excite the probe, and an MCP photomultiplier (Hamamatsu R3809U-50) was used as the detector (response time 40 ps). The excitation source profile was recorded by scatterer

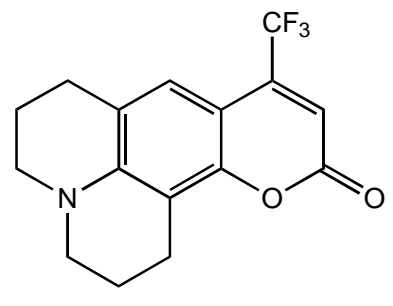

Coumarin 153 (C153)

Figure 1: Molecular Structures of Bis(1-methyl-1H-imidazol-3-ium-3-yl)dihydroborate bis(trifluoromethylsulfonyl)amide [BIMIMDBA][ NTf 2 and Coumarin153 (C153). 
(dilute ludox solution in water) in place of the sample. The temperature was controlled by the Quantum Northwest (TC125) instrument. Decay curves were analyzed by nonlinear least-squares iteration procedure using F900 decay analysis software. The quality of the fit was judged by the chi square $\left(\chi^{2}\right)$ values and weighted deviation was obtained by fitting. The time-resolved fluorescence anisotropy measurement was done by using two polarizer by placing one of them in the excitation beam path and the other one in front of the detector. An alternate collection of the fluorescence intensity in parallel and perpendicular polarization (with respect to the vertically polarized excitation laser beam) for equal interval of time had been carried out until the count difference between the two polarizations (at $t=0$ ) is $\sim 5000$. For G-factor calculation, the same procedure was adopted, but with 5 cycles and horizontal polarization of the exciting laser beam.

\section{Method}

The time resolved emission decay profile were measured at $5 / 10 \mathrm{~nm}$ intervals around the whole emission spectra. The total number of measurements was 25-35 in each case. In all cases, when decay was monitored at a shorter wavelength, a faster decay was observed, and at a longer wavelength, we observed a decay profile that consists clear rise with usual decay. Each decay curve was fitted to a triexponential decay function with an iterative reconvolution program (F900) maintaining the $\chi^{2}$ between 1 and 1.2 as a measure of goodness of fit. The time resolved emission spectra (TRES) were constructed according to a procedure described earlier [13].

The emission maximum at each time $\bar{v}(t)$ was obtained by fitting the spectrum to a log-normal lineshape function as given below

$I=h \exp \left[-\ln 2\{\ln (1+\alpha) / y\}^{2}\right]$

for $\alpha>-1$ and $I=0$ for $\alpha \leq-1$

where $\alpha=2 \gamma\left(\bar{v}-\bar{v}_{\text {peak }}\right) / \Delta, \bar{v}_{\text {peak }}$ was the wavenumber corresponding to the peak, $h$ was the peak height, $\Delta$ was the full width at half maximum (FWHM) and $y$ was a measure of the asymmetry of the band shape. Optimizing these four parameters by nonlinear least squares iteration, the best fitted curve was obtained. The peak frequencies were obtained from this lognormal fitting of TRES. The peak frequencies were then used to construct the solvent correlation function $(C(t))$.
$C(t)=\frac{\bar{v}(t)-\bar{v}(\infty)}{\bar{v}(0)-\bar{v}(\infty)}$

where $\bar{v}(0)$ was the peak frequency at time $t=0$, just after the electronic excitation and $\bar{v}(t)$ was the peak frequency at time $t=t$. Again, $\bar{v}(\infty)$ was the peak frequencies at $t=\infty$ when solvent molecules were in the equilibrium position around the photoexcited probe molecule. The time dependence of the calculated $C(t)$ was fitted by a biexponential function of the form

$C(t)=a_{1} e^{-t / \tau}{ }_{1}+a_{2} e^{-t / \tau} \tau_{2}$

where $\tau_{1}$ and $\tau_{2}$ are the solvent relaxation times and $\mathrm{a}_{1}$ and $\mathrm{a}_{2}$ were normalized pre-exponential factors. After having the values of $\tau_{1}, \tau_{2}$ and $a_{1}, a_{2}$, the average solvation time was calculated by using the following relation

$<\tau_{\text {solv. }}>=a_{1} \tau_{1}+a_{2} \tau_{2}$

We have also fitted $C(t)$ by the stretched exponential function as given below.

$C(t)=\exp \left(-\left(t / \tau_{\text {solv }}\right)^{\beta}\right)$

Where $0<\beta \leq 1$

$\left\langle\tau_{s t}\right\rangle=\frac{\tau_{s o l v}}{\beta} \Gamma\left(\beta^{-1}\right)$

where $\Gamma$ was the gamma function and $\tau_{s t}$ was the average solvation time considering $C(t)$ was a stretched exponential function.

\section{RESULTS AND DISCUSSION}

\section{Optimized Structure}

To know the structural features of [BIMIMDBA] $\left[\mathrm{TF}_{2} \mathrm{~N}\right]$ we have optimized the structure of this IL in the ground state with B3LYP functional [39, 40] and $6-31++\mathrm{G}(\mathrm{d}, \mathrm{p})$ basis set using Gaussian 3.0 program [41]. The optimized structure of $[\mathrm{BIMIMDBA}]\left[\mathrm{TF}_{2} \mathrm{~N}\right]$ is shown in Figure 2. The van der Waals criterion for hydrogen bond formation is that for the formation of $\mathrm{X}-\mathrm{H} \cdots \mathrm{Z}$ bonds, the distance between $X \cdots Z$ should be less than the sum of the $X-H$ covalent bond and the van der Waals radii of $H$ and $Z$ [42]. The van der Waals radii of $\mathrm{O}, \mathrm{N}$ and $\mathrm{H}$ are $1.52 \AA$, $1.55 \AA$ and $1.2 \AA$ respectively. In the optimized structure, the distance between $\mathrm{O} 33-\mathrm{H} 17, \mathrm{O} 34-\mathrm{H} 4$ and $\mathrm{N} 28-\mathrm{H} 17$ are measured to be $2.614 \AA$, $2.172 \AA$ and $2.451 \AA$ respectively (Figure 2 ). Moreover, the bond angles 


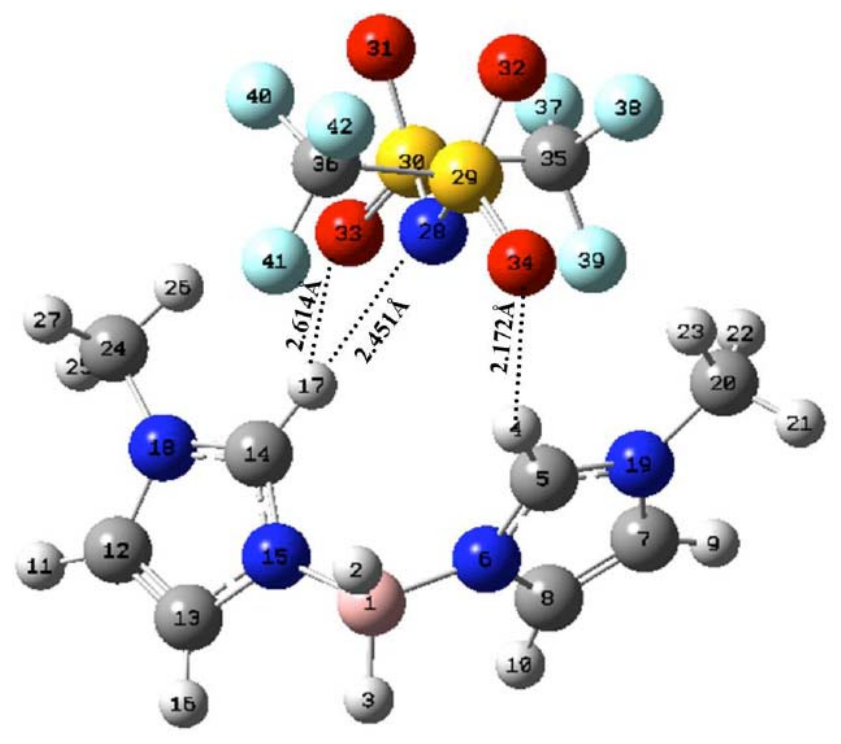

Figure 2: Optimized structure of [BIMIMDBA][TF $2 \mathrm{~N}]$.

$\mathrm{C} 14-\mathrm{H} 17-\mathrm{N} 28, \mathrm{C} 14-\mathrm{H} 17-\mathrm{O} 33$ and $\mathrm{C} 5-\mathrm{H} 4-\mathrm{O} 34$ are found to be $179.4^{\circ}, 122.3^{\circ}$ and $141.3^{\circ}$ respectively. In the present case, these calculated data of both the distance and the angle suggests the presence of strong $\mathrm{C}-\mathrm{H} \cdots \mathrm{N}$ hydrogen bonding interactions between the cationic and the anionic fragment of the RTIL. The data also depicts a weak $\mathrm{C}-\mathrm{H} \cdots \mathrm{O}$ interaction between the cationic and the anionic moiety. The structural parameters as obtained from the gas phase optimized structure of the present ionic liquid are collected in Table 1.

\section{Steady State Behavior}

The absorption and emission spectra of $\mathrm{C} 153$ are recorded both in neat RTIL and RTIL-DMF mixture.
The absorption and emission maxima of $\mathrm{C} 153$ in neat ionic liquid and its binary mixture with DMF are shown in Table 2. The representative steady state emission spectra of C153 in neat RTIL and RTIL-DMF binary mixtures are also shown in Figure 3 . In the present case, no significant emission from the ionic liquid has been observed; the fact has enabled us to use the present ionic liquid for photophysical studies. Interestingly, the position of the absorption and emission maximum of $\mathrm{C} 153$ remain unchanged after the addition of organic cosolvent to RTIL.

\section{Time-Resolved Studies}

The emission decay profile of $\mathrm{C} 153$ has been measured at several wavelengths (5-10 nm intervals) across the emission spectra by exciting the sample at $405 \mathrm{~nm}$. The decay profiles are found to be strongly dependent on the monitoring wavelengths (Figure 4). A faster decay is observed when the process is monitored at shorter wavelengths. However, at longer wavelengths, we observed a decay profile that consists of a clear rise with usual decay. This particular observation carries the typical signature of solvation process [38]. The time-resolved emission spectra (TRES) of the system, constructed from the fitted decay profiles, show progressive red shift of the fluorescence maximum with time. Representative TRES of C153 is shown in Figure 5. Time-dependent shifts of the emission maximum to lower energy have been observed in all cases. This indicates that the excited states of dipolar molecules are stabilized by solvent molecules with time.

Table 1: Bond Lengths and Bond Angle in Optimized Structure of [BIMIMDBA][TF 2 ] Obtained from B3LYP Functional/6-31++G (d, p) Level Optimization

\begin{tabular}{|c|c|c|c|c|}
\hline Systems & Gas phase distances & $(\AA ̊ \cap)$ & Gas phase bond angles & $\left({ }^{\circ}\right)$ \\
\hline \multirow[t]{12}{*}[\mathrm{BIMIMDBA}]{$\left[\mathrm{TF}_{2} \mathrm{~N}\right]$} & $\mathrm{C} 5-\mathrm{H} 4$ & 1.081 & C14-H17-N28 & 179.40 \\
\hline & $\mathrm{C} 14-\mathrm{H} 17$ & 1.083 & $\mathrm{C} 14-\mathrm{H} 17-\mathrm{O} 33$ & 122.30 \\
\hline & $\mathrm{N} 28-\mathrm{H} 4$ & 3.034 & $\mathrm{C} 5-\mathrm{H} 4-\mathrm{O} 34$ & 141.30 \\
\hline & $\mathrm{N} 28-\mathrm{H} 17$ & 2.451 & $\mathrm{C} 14-\mathrm{H} 17-\mathrm{O} 34$ & 143.10 \\
\hline & $\mathrm{O} 34-\mathrm{H} 4$ & 2.172 & \multirow[t]{8}{*}{ C14-H4-N28 } & \multirow[t]{8}{*}{100.04} \\
\hline & O33-H17 & 2.614 & & \\
\hline & F39-H22 & 2.726 & & \\
\hline & F39-H23 & 3.825 & & \\
\hline & $\mathrm{F} 41-\mathrm{H} 26$ & 4.585 & & \\
\hline & C14-H17-N28 & 3.833 & & \\
\hline & $\mathrm{C} 14-\mathrm{H} 17-\mathrm{O} 33$ & 3.800 & & \\
\hline & $\mathrm{C} 5-\mathrm{H} 4-\mathrm{O} 34$ & 3.800 & & \\
\hline
\end{tabular}


Table 2: Absorption and Emission Maxima, Stokes Shift of C153 in Different Systems

\begin{tabular}{|c|c|c|c|}
\hline System & $\lambda_{\max }(\mathbf{a b s})[\mathrm{nm}]$ & $\boldsymbol{\lambda}_{\max }(\mathbf{e m})[\mathrm{nm}]$ & Stokes Shift(cm $\left.^{-1}\right)$ \\
\hline \hline RTIL+0\%DMF & 442 & 528 & 3685 \\
\hline $\mathrm{RTIL+6 \% DMF}$ & 442 & 528 & \\
\hline $\mathrm{RTIL}+12 \% \mathrm{DMF}$ & 442 & 528 & \\
\hline
\end{tabular}

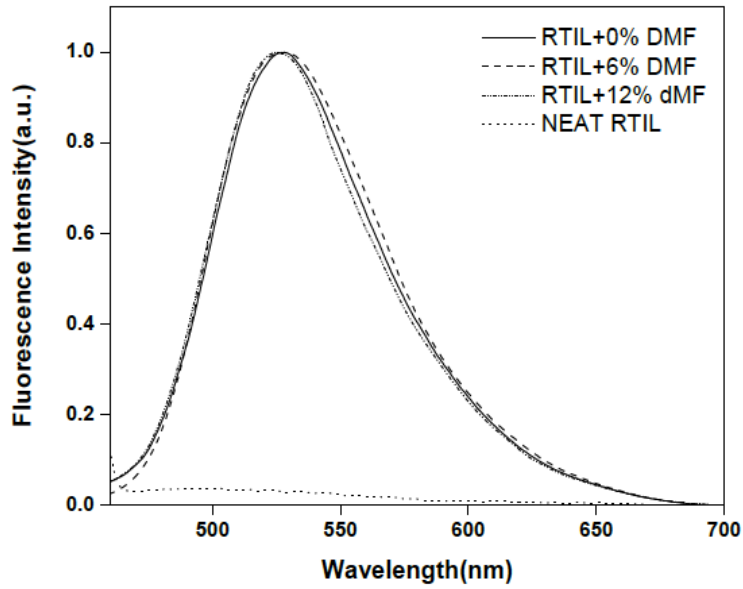

Figure 3: Normalised steady state emission spectrum of C153 in neat RTIL+ DMF mixtures. Emission spectrum of neat RTIL is also shown in the same figure. $\lambda_{\text {exc. }}=405 \mathrm{~nm}$.
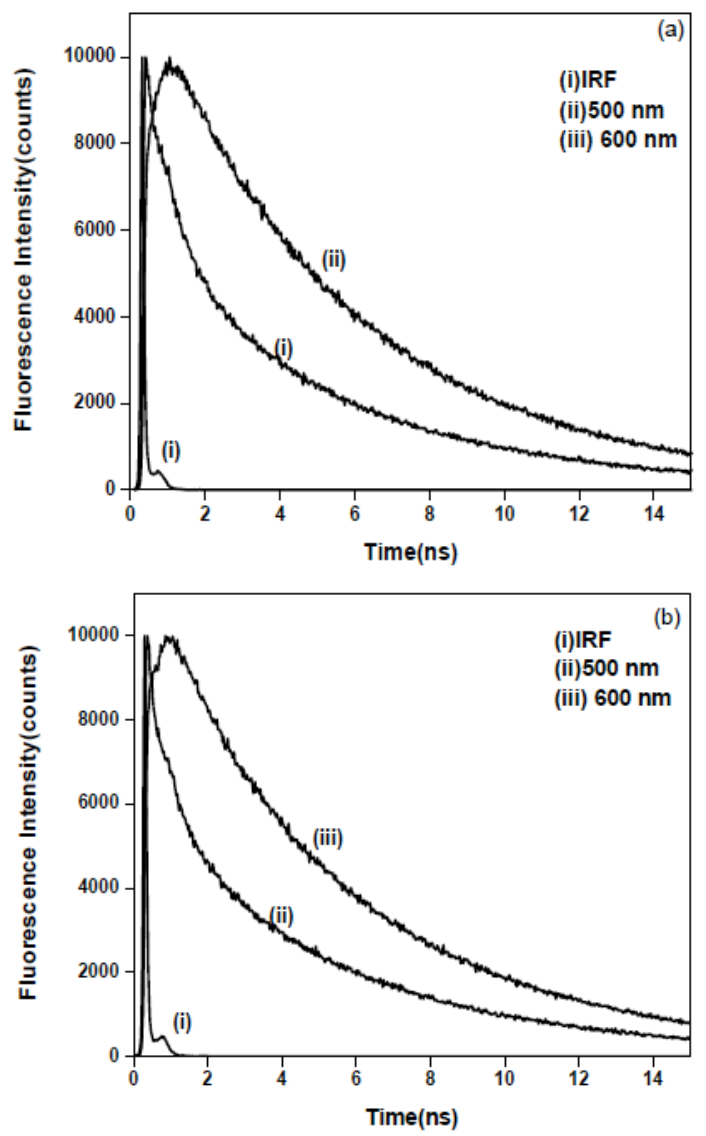

Figure 4: Decay profiles of $\mathrm{C} 153$ at different monitoring wavelengths in (a) RTIL+ 6\% DMF and in (b) RTIL+12\% DMF.
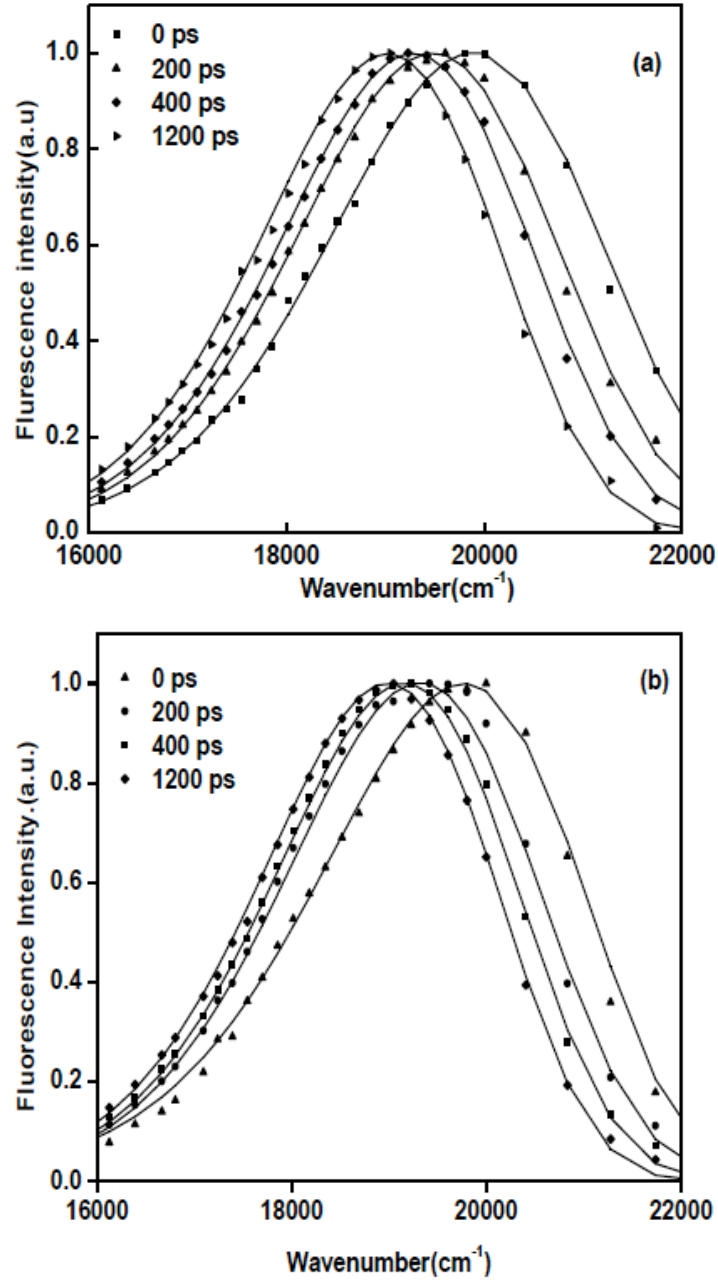

Figure 5: TRES plots $\mathrm{C} 153$ in (a) neat RTIL and in (b) RTIL+6wt\% DMF mixture.

The total Stokes shift of the fluorescence maximum observed and the relaxation parameters of solvation of C153 for the systems are collected in Table 3. The time constants of the observable dynamics are determined from the plots of the spectral shift correlation function, $C(t)$, versus time. Average solvation time has been calculated by using both bi-exponential function and stretched exponential function. We found that both the fits are providing almost same average solvation time. Decay of solvation correlation function, $C(t)$ of $C 153$ in neat RTIL and RTIL-DMF binary mixtures are shown in the Figure 6. 
Table 3: Solvation Relaxation Parameters of C153 in RTIL-DMF Binary Mixtures

\begin{tabular}{|c|c|c|c|c|c|c|c|c|c|c|}
\hline \multirow{2}{*}{$\begin{array}{l}\text { Wt\% } \\
\text { DMF }\end{array}$} & \multirow[t]{2}{*}{${ }^{\mathrm{a}}$ Viscosity(cP) } & \multicolumn{5}{|c|}{${ }^{b}$ Biexponential Fit } & \multicolumn{3}{|c|}{${ }^{\mathrm{c}}$ Stretched Exponential Fit } & \multirow{2}{*}{$\begin{array}{c}\text { Observed Shif } \\
\left(\mathrm{cm}^{-1}\right)\end{array}$} \\
\hline & & $a_{1}$ & $\tau_{1}(n s)$ & $a_{2}$ & $\tau_{2}(n s)$ & $\tau_{\text {avg }}(n s)$ & $\beta$ & $\tau_{\text {solv }}$ & $\left\langle\tau_{\mathrm{st}}\right\rangle(\mathbf{n s})$ & \\
\hline 0 & 160 & 0.57 & 0.23 & 0.42 & 1.11 & 0.6 & 0.74 & 0.47 & 0.57 & 1016 \\
\hline 6 & 78 & 0.64 & 0.17 & 0.36 & 0.84 & 0.41 & 0.738 & 0.317 & 0.38 & 855 \\
\hline 12 & 40.7 & 0.83 & 0.14 & 0.18 & 0.67 & 0.24 & 0.841 & 0.194 & 0.21 & 910 \\
\hline
\end{tabular}

${ }^{\mathrm{a}}$ at $298 \mathrm{~K},{ }^{\mathrm{b}}$ by equation (4), ${ }^{\mathrm{c}}$ by equation (6), ${ }^{\mathrm{d}}$ calculated by $\left[\bar{v}_{o}-\bar{v}_{\infty}\right]$

From Table 3, it is evident that the average solvation time of $\mathrm{C} 153$ decreases with the increase in wt. percentage of DMF in RTIL. For example, the average solvation time decreases from $600 \mathrm{ps}$ in neat ionic liquid to $240 \mathrm{ps}$ in RTIL-DMF mixture (12 wt\%). This lowering of average solvation time with gradual addition of DMF to neat ionic liquid could be attributed to gradual lowering of bulk viscosity of the media. To get a better understanding on the dependence of average solvation time on bulk viscosity of the probe molecule in the different RTIL-DMF composition, we have plotted the average solvation time of C153 against the bulk viscosity values of RTIL-DMF mixtures (Figure 7). Almost a linear decrease in average solvation time with bulk viscosity has been observed. In this context we would like to note that in neat DMF the average solvation and rotational time are found to be 2 ps and 47 ps respectively [43,44]. The faster dynamics is attributed to the much low viscosity of DMF.

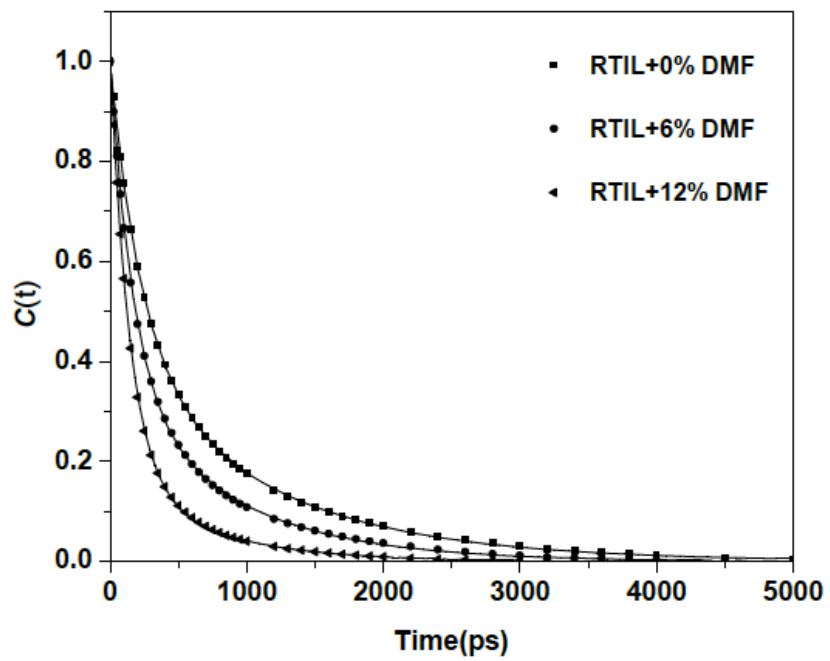

Figure 6: Decay of the spectral shift correlation function, $C(t)$ of $\mathrm{C} 153$ in neat RTIL, RTIL+6wt\% DMF and RTIL+12wt\% DMF $\left(\lambda_{\text {exc. }}=405 \mathrm{~nm}\right)$. In all cases, solid lines denote the biexponential fit to the data points.

Time-resolved fluorescence anisotropy $(r(t))$ is estimated by the following equation

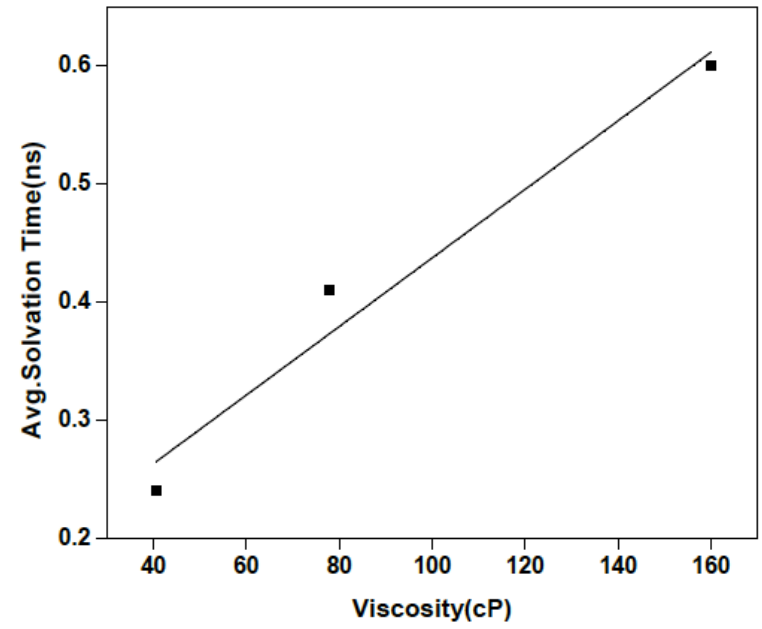

Figure 7: Average solvation time against bulk viscosity at $293 \mathrm{~K}$.

$r(t)=\frac{G I_{V V}(t)-I_{V H}(t)}{G I_{V V}(t)+2 I_{V H}(t)} \quad$ Where $G=\frac{\sum I_{H H}(t)}{\sum I_{H V}(t)}$

where $G$ is the correction factor for detector sensitivity to the polarization of the emission. $G$ is 0.8 at the wavelength of detection. $I_{H H}(t)$ and $I_{H V}(t)$ are the intensities of fluorescence decays when the excitation and the emission polarizers are polarized at horizontalhorizontal and horizontal-vertical alignments respectively. Again $I_{V V}(t)$ and $I_{V H}(t)$ are the intensities of fluorescence decays when excitation and emission polarizers are polarized at vertical-vertical and vertical-horizontal alignments respectively. The rotational relaxation parameters of $\mathrm{C} 153$ in neat RTIL and in RTIL-DMF at different temperatures are collected in Table 4. The anisotropy decay profiles are fitted to a bi-exponential function.

Decay of time-resolved anisotropy $(r(t))$ of $\mathrm{C} 153$ in $\mathrm{RTIL}+6 \%$ (wt.) DMF at various temperatures are shown in Figure 8.

As can be seen from Table 4, the average rotational time of $\mathrm{C} 153$ which is higher in neat ionic liquid 
Table 4: Rotational Relaxation Parameters of C153 in RTIL-DMF Binary Mixtures at Different Temperatures $\left(\lambda_{\text {exc }}=405\right.$ $\mathrm{nm})$

\begin{tabular}{|c|c|c|c|c|c|c|c|c|c|}
\hline $\begin{array}{l}\text { Wt\% of DMF } \\
\text { in RTIL }\end{array}$ & Temp.(K) & Viscosity & ${ }^{a} \mathbf{r}_{0}$ & $a_{1}$ & $\begin{array}{c}\tau_{1} \\
\text { (ns) }\end{array}$ & $a_{2}$ & $\begin{array}{c}\tau_{2} \\
\text { (ns) }\end{array}$ & $\begin{array}{l}<\tau_{r}> \\
\text { (ns) }\end{array}$ & ${ }^{\mathrm{b}} \mathrm{C}_{\text {rot }}$ (avg.) \\
\hline \multirow[t]{5}{*}{0} & 293 & 160 & 0.35 & 0.17 & 0.75 & 0.83 & 7.69 & 6.52 & \multirow[t]{5}{*}{0.48} \\
\hline & 298 & 117.4 & 0.36 & 0.19 & 0.47 & 0.81 & 5.72 & 4.74 & \\
\hline & 303 & 88.5 & 0.35 & 0.17 & 0.53 & 0.82 & 4.50 & 3.82 & \\
\hline & 308 & 68.3 & 0.35 & 0.18 & 0.48 & 0.82 & 3.52 & 2.95 & \\
\hline & 313 & 53.5 & 0.35 & 0.18 & 0.31 & 0.82 & 2.72 & 2.29 & \\
\hline \multirow[t]{5}{*}{6} & 293 & 78 & 0.32 & 0.22 & 1.11 & 0.77 & 5.37 & 4.41 & \multirow[t]{5}{*}{0.59} \\
\hline & 298 & 62.3 & 0.32 & 0.19 & 0.83 & 0.80 & 3.95 & 3.35 & \\
\hline & 303 & 50.7 & 0.30 & 0.20 & 0.74 & 0.80 & 3.19 & 2.70 & \\
\hline & 308 & 42.2 & 0.30 & 0.18 & 0.52 & 0.82 & 2.40 & 2.06 & \\
\hline & 313 & 35.8 & 0.30 & 0.23 & 0.72 & 0.77 & 2.07 & 1.75 & \\
\hline \multirow[t]{5}{*}{12} & 293 & 40.7 & 0.32 & 0.23 & 0.85 & 0.76 & 3.14 & 2.6 & \multirow[t]{5}{*}{0.62} \\
\hline & 298 & 32.3 & 0.32 & 0.20 & 0.57 & 0.80 & 2.36 & 2.00 & \\
\hline & 303 & 26 & 0.38 & 0.27 & 0.09 & 0.72 & 1.68 & 1.24 & \\
\hline & 308 & 23.5 & 0.35 & 0.20 & 0.13 & 0.80 & 1.41 & 1.15 & \\
\hline & 313 & 19.7 & 0.36 & 0.20 & 0.13 & 0.80 & 1.17 & 0.95 & \\
\hline
\end{tabular}

${ }^{a} r_{0}$ is the initial anisotropy.

${ }^{b} \mathrm{C}_{\text {rot }}$ is rotational coupling constant calculated according to equation 11. Temperature averaged $\mathrm{C}_{\text {rot }}$ values are tabulated.

gradually decreases with gradual addition of DMF to RTIL. For example, at $293 \mathrm{~K}$, the average rotational time of $\mathrm{C} 153$ decreases from $6.52 \mathrm{~ns}$ in neat ionic liquid to $2.60 \mathrm{~ns}$ in RTIL-DMF (12wt \%) mixture. The faster rotation of $\mathrm{C} 153$ with decrease in viscosity of the RTIL-DMF mixture is also evident when the average rotational time of $\mathrm{C} 153$ is plotted against the viscosity of the media. The log-log plot of average rotational relaxation time $\left(\tau_{r}\right)$ against viscosity with respect to temperature is shown in Figure 9. It is clear from the plot that rotational relaxation dynamics of $\mathrm{C} 153$ can be well explained within the broad limits of SED hydrodynamic theory. It is also evident from the plot that the average rotational relaxation time decreases with lowering of the bulk viscosity of the media. We have also fitted the $\left\langle\tau_{r}>\right.$ and $\eta / T$ data for $\mathrm{C} 153$ to the function, $\tau_{r}=A(\eta / T)^{P}$. In these expressions (equations $8,9$ and 10$)$, the $\tau_{r}$ values are in nanoseconds and $\eta / T$ are in $\mathrm{CPK}^{-1}$, and $N$ and $R$ denote the number of data points and regression coefficient, respectively. Prominent nonlinear composition dependence has not been observed in the present case (equations 8, 9 and 10).

C153 in RTIL+ 0(wt\%)DMF

$<\tau_{r}>=(11.203 \pm 0.2913)(\eta / T)^{0.887 \pm 0.02584}(\mathrm{~N}=5$,

$\mathrm{R}=0.9988$ )

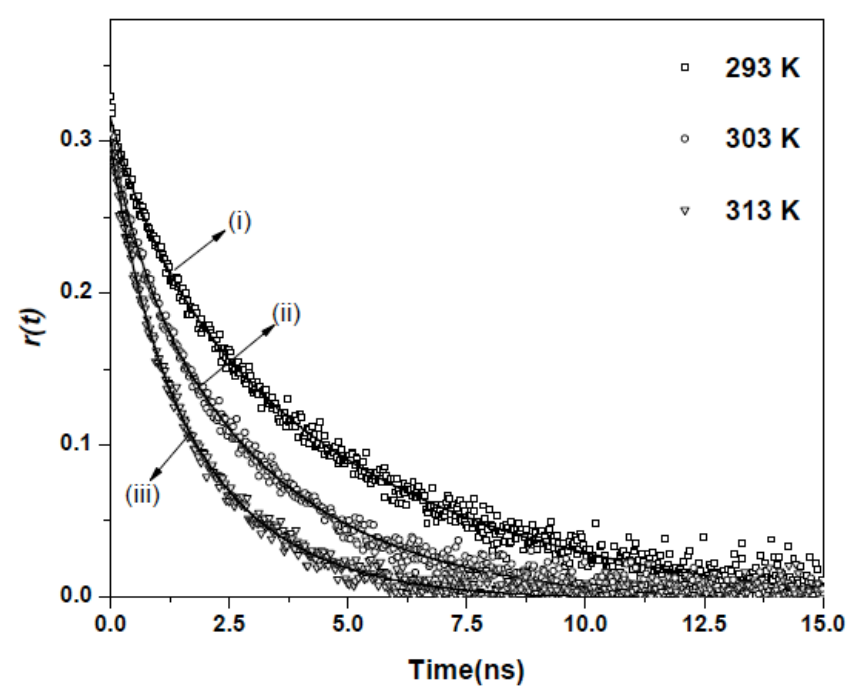

Figure 8: Decay of time-resolved anisotropy $(r(t))$ of $\mathrm{C} 153$ in $\mathrm{RTIL}+6 \%$ (wt.) DMF at $\lambda_{\text {exc. }}=405 \mathrm{~nm}$ at temperatures (i) 293 $\mathrm{K}$, (ii) $303 \mathrm{~K}$ and (iii) $313 \mathrm{~K}$ respectively. Solid line represents biexponential fitting to the data points.

C153 in RTIL+ 6(wt\%)DMF

$<\tau_{r}>=(18.704 \pm 0.7959)(\eta / T)^{1.069 \pm 0.026}(\mathrm{~N}=5, \mathrm{R}=0.9991)$

C153 in RTIL+ 12(wt\%)DMF

$<\tau_{r}>=(32.9689 \pm 13.62)(\eta / T)^{1.28 \pm 0.185}(\mathrm{~N}=5, \mathrm{R}=0.9712)$ 


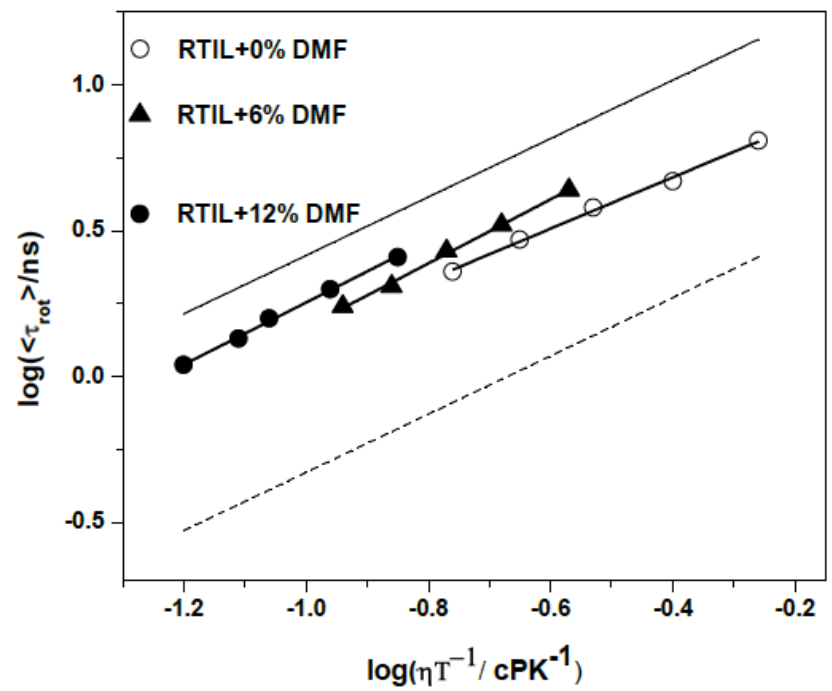

Figure 9: The log-log plots of $\left\langle\tau_{r}\right\rangle$ Vs. $(\eta / T)$ showing the stick and slip boundary conditions with solid and dashed line respectively.

We have also estimated the rotational coupling constants $\left(C_{\text {rot }}\right)$ of $\mathrm{C} 153$ at different RTIL-DMF composition. According to the stick hydrodynamic theory, the rotational time constant $\left(\tau_{s t k}\right)$ of a nonspherical solute of volume $V$, rotating along the axis of the ellipsoid in a medium of viscosity $\eta$ at temperature $T$ is given by

$\tau_{s t k}=V f_{s t k} \eta / k_{B} T$

where $f_{\text {stk }}$ accounts for nonspherical shape of the solute and $k_{B}$ is the Boltzmann constant. Using the literature value of $V\left(243 \AA^{3)}\right.$ and $f_{s t k}(1.5)$, we computed the $\tau_{\text {stk }}$ values for RTIL and RTIL-DMF mixture [7]. From the computed $\tau_{\text {stk }}$ values, the rotational coupling constants, $C_{\text {rot }}$, which is defined as $C_{\text {rot }}=\tau_{\text {rot }} \tau_{\text {stk }}$, is calculated. As can be seen from Table 4 , the $C_{\text {rot }}$ value varies from 0.48 to 0.62 . The calculated $C_{\text {rot }}$ values, which are measure of the extent of departure from normal hydrodynamic behavior of a solute due to specific interaction, are found to be very similar to that obtained for C153 in conventional solvents [44]. The calculated $C_{\text {rot }}$ values also indicate no involvement of specific solute-solvent interaction in the present case. The observation demonstrates that other than the loweing of viscosity of the medium, DMF does not seem to play any role towards the rotational dynamics of $\mathrm{C} 153$ in RTIL-DMF mixtures.

\section{CONCLUSION}

We have studied the steady state and time resolved fluorescence behavior of C153 in a new diimidazolium cation based ionic liquid and its mixture with organic polar cosolvent DMF to determine the role of DMF that it plays in governing the solvation and rotational relaxation of $\mathrm{C} 153$ in these media. The present ionic liquid has negligible emission behavior and so is well suited for fluorescence studies. Steady state spectral behavior of $\mathrm{C} 153$ is found not to be affected by these polar cosolvents. However, in time-resolved fluorescence studies the influence of DMF is clearly observed. The addition of DMF to RTIL decreases the average solvation time due to a lowering of viscosity of the media. This also results in the faster rotational dynamics of $\mathrm{C} 153$.

\section{ACKNOWLEDGEMENT}

MS is thankful to Department of Science and Technology (DST), Government of India, for a research grant. PKS thanks National Institute of Science Education and research (NISER) and SKD thanks Council of Scientific and Industrial Research (CSIR) for the fellowship.

\section{REFERENCES}

[1] Wasserscheid P, Keim W. Ionic liquids: New "solutions" for transition metal catalysis. Angew Chem Int Ed 2000; 39: 3772-89.

http://dx.doi.org/10.1002/1521-

$3773(20001103) 39: 21<3772::$ AID-ANIE3772>3.0.CO:2-5

[2] Rogers RD, Seddon KR. Ionic Liquids-Solvents of the future? Science 2003; 302: 792-3. http://dx.doi.org/10.1126/science.1090313

[3] Köddermann T, Wertz C, Heintz A, Ludwig R. The association of water in ionic liquids: A reliable measure of polarity. Angew Chem Int Ed 2006; 45: 3697-70. http://dx.doi.org/10.1002/anie.200504471

[4] Samanta A. Dynamic Stokes shift and excitation wavelength dependent fluorescence of dipolar molecules in room temperature ionic liquids. J Phys Chem B 2006; 110: 137046.

http://dx.doi.org/10.1021/jp060441d

[5] Samanta A. Solvation dynamics in ionic liquids: What we have learned from the dynamic fluorescence Stokes shift studies. J Phys Chem Lett 2010; 1(10): 1557-62. http://dx.doi.org/10.1021/jz100273b

[6] Paul A, Samanta A. Effect of nonpolar solvents on the solute rotation and solvation dynamics in an imidazolium ionic liquid. J Phys Chem B 2008; 112: 947-53. http://dx.doi.org/10.1021/jp077536s

[7] Ito N, Arzhantsev S, Maroncelli M. The probe dependence of solvation dynamics and rotation in the ionic liquid 1-butyl-3methyl-imidazolium hexafluorophosphate.Chem Phys Lett 2004; 396: 83-91.

http://dx.doi.org/10.1016/j.cplett.2004.08.018

[8] Chakrabarty D, Chakraborty D, Seth D, Hazra P, Sarkar N. Dynamics of solvation and rotational relaxation of Coumarin 153 in 1-butyl-3-methylimidazolium hexafluorophosphate [bmim] $\left[\mathrm{PF}_{6}\right]$-water mixtures. Chem Phys Lett 2004; 397; 469-74. http://dx.doi.org/10.1016/i.cplett.2004.08.141

[9] Chakrabarty D, Chakraborty D, Seth D, Hazra P, Sarkar N. Effect of Water, Methanol, and Acetonitrile on Solvent 
Relaxation and Rotational Relaxation of Coumarin 153 in Neat 1-Hexyl-3-methylimidazolium Hexafluorophosphate. J Phys Chem A 2005; 109; 1764-69.

http://dx.doi.org/10.1021/ip0460339

[10] Pramanik R, Rao VG, Sarkar S, Ghatak C, Setua P, Sarkar $\mathrm{N}$. To probe the interaction of methanol and acetonitrile with the ionic liquid $N, N, N$-Trimethyl- $N$-propyl ammonium Bis(trifluoromethanesulfonyl) imide at different temperatures by solvation dynamics study. J Phys Chem B 2009; 113: 8626-34.

http://dx.doi.org/10.1021/jp900627h

[11] Sarkar S, Pramanik R, Ghatak C, Setua P, Sarkar N. Probing the interaction of 1-Ethyl-3-methylimidazolium Ethyl Sulfate $\left([\mathrm{Emim}]\left[\mathrm{EtSO}_{4}\right]\right)$ with alcohols and water by solvent and rotational relaxation. J Phys Chem B 2010; 114: 2779-89. http://dx.doi.org/10.1021/jp907936s

[12] Daschakraborty S, Biswas R. Stokes shift dynamics in (ionic liquid + polar solvent) binary mixtures: Composition dependence. J Phys Chem B 2011; 115: 4011-24. http://dx.doi.org/10.1021/jp200407m

[13] Das SK, Sarkar M. Steady-state and time-resolved fluorescence behavior of coumarin-153 in a hydrophobic ionic liquid and ionic liquid-toluene mixture. J Mol Liq 2012; 165: 38-43.

http://dx.doi.org/10.1016/j.molliq.2011.10.004

[14] Gutowski KE, Broker GA. Willauer HD, et al. Controlling the aqueous miscibility of ionic liquids: Aqueous biphasic systems of water-miscible ionic liquids and water-structuring salts for recycle, metathesis, and separations. J Am Chem Soc 2003; 125: 6632-3. http://dx.doi.org/10.1021/ja0351802

[15] Dupont J, de Suza RF, Suarez PAZ. Ionic liquid (molten salt) phase organometallic catalysis. Chem Rev 2002; 102: 366792.

http://dx.doi.org/10.1021/cr010338r

[16] GZ Wu, Liu YD, Long DW. Effect of ionic liquid $\left[\mathrm{Me}_{3} \mathrm{NC}_{2} \mathrm{H}_{4} \mathrm{OH}\right]^{+}\left[\mathrm{ZnCl}_{3}\right]^{-}$on $\mathrm{Y}$ - radiation polymerization of methylmethacrylate in ethanol and N,N-Dimethylformamde. Macromol Rapid Commun 2005; 26: 57-9.

http://dx.doi.org/10.1002/marc.200400375

[17] Dai S, Ju YH, Barnes CE. Solvent extraction of strontium nitrate by a crown ether using room-temperature ionic liquids. Dalton Trans 1999; 1201-2.

[18] Visser AE, Swatloski RP, Reichert WM, Griffin ST, Rogers RD. Traditional extractants in nontraditional solvents: Groups 1 and 2 extraction by crown ethers in room-temperature ionic liquids. Ind Eng Chem Res 2000; 29: 3596-604. http://dx.doi.org/10.1021/ie000426m

[19] Widegen JA, Laesecke A, Magee JW. The effect of dissolved water on the viscosities of hydrophobic room temperature ionic liquids. Chem Commun 2005; 1610-2.

http://dx.doi.org/10.1039/b417348a

[20] Fletcher KA, Pandey S. Solvatochromic probe behavior within ternary room-temperature ionic liquid 1-Butyl-3methylimidazolium Hexafluorophosphate + ethanol + water solutions. J Phys Chem B 2003; 107: 13532-9. http://dx.doi.org/10.1021/jp0276754

[21] Baker SN, Baker GA, Bright FV. Temperature-dependent microscopic solvent properties of 'dry'and 'wet' 1-butyl-3methylimidazolium hexafluorophosphate: correlation with $\mathrm{E}_{\mathrm{T}}(30)$ and Kamlet-Taft polarity scales. Green Chem 2002; 4: $165-9$.

http://dx.doi.org/10.1039/b111285f

[22] Fletcher KA, Pandey S. Solvatochromic probe behavior within binary room-temperature ionic liquid 1-Butyl-3-methyl imidazolium Hexafluorophosphate plus ethanol solutions. Appl Spectrosc 2002; 56: 1498-504. http://dx.doi.org/10.1366/00037020260377823
[23] Harifi-Mood AR, Habibi-Yangjeh A, Gholami MR. Solvatochromic parameters for binary mixtures of 1-(1-Butyl)3-methylimidazolium Tetrafluoroborate with some protic molecular solvents. J Phys Chem B 2006; 110: 7073-8.

http://dx.doi.org/10.1021/jp0602373

[24] Mellein BR, Aki SNVK, Ladewski RL, Brennecke JF. Effect of water and organic solvents on the ionic dissociation of ionic liquids. J Phys Chem B 2007; 111: 6452-6. http://dx.doi.org/10.1021/jp071051m

[25] Jarosik A, Krajewski SR, Lewandowski A, Radzimski P. Conductivity of ionic liquids in mixtures. J Mol Liq 2006; 123 : 43-50. http://dx.doi.org/10.1016/j.molliq.2005.06.001

[26] Tokuda H, Baek SJ, Watanable M. Room-temperature ionic liquid-organic solvent mixture: Conductivity and ion association. Electrochemistry 2005; 73: 620-2.

[27] Žagar E, Žigon M. Solution properties of carboxylated polyurethanes and related ionomers in polar solvents (DMF and LiBr/DMF). Polymer 2000; 41: 3513-21. http://dx.doi.org/10.1016/S0032-3861(99)00604-7

[28] Kang YK, Park HS. Internal rotation about the $\mathrm{C}-\mathrm{N}$ bond of amides. J Mol Struct (Theochem) 2004; 676: 171-6. http://dx.doi.org/10.1016/j.theochem.2004.01.024

[29] Borrmann H, Persson I, Sandström M, Stålhandske CMV. The crystal and liquid structures of $\mathrm{N}, \mathrm{N}$ dimethylthioformamide and $N, N$-dimethylformamide showing a stronger hydrogen bonding effect for $\mathrm{C}-\mathrm{H}$...S than for CH...O. J Chem Soc Perkin Trans 2 2000; 393-402. http://dx.doi.org/10.1039/a904531g

[30] Umebayashi $\mathrm{Y}$, Matsumoto $\mathrm{K}$, Watanabe $\mathrm{M}$, Ishiguro $\mathrm{S}$. Individual solvation number of first-row transition metal(II) ions in solvent mixtures of $\mathrm{N}, \mathrm{N}$-dimethylformamide and $\mathrm{N}, \mathrm{N}$ dimethylacetamide-Solvation steric effect. Phys Chem Chem Phys 2001; 3: 5475-81.

http://dx.doi.org/10.1039/b107342g

[31] García B, Alcalde R, Leal JM, Matos JS. Shear viscosities of the N-methylformamide- and N,N-dimethylformamide- $\left(\mathrm{C}_{1}-\right.$ $\mathrm{C}_{10}$ ) alkan-1-ol solvent systems. J Chem Soc Faraday Trans 1997; 93: 1115-8.

http://dx.doi.org/10.1039/a607876a

[32] Venkatesu P, Lee MJ, Lin HM. Volumetric properties of $(\mathrm{N}, \mathrm{N}$ dimethylformamide + aliphatic diethers) at temperatures ranging from (298.15 to 358.15 ) K. J Chem Thermodyn 2005; 53: 996-1002. http://dx.doi.org/10.1016/j.jct.2005.01.002

[33] Attri P, Reddy PM, Venkatesu P, Kumar A, Hofman T. Measurements and molecular interactions for $\mathrm{N}, \mathrm{N}$ Dimethylformamide with ionic liquid mixed solvents. J Phys Chem B 2010; 114: 6126-33. http://dx.doi.org/10.1021/jp101209j

[34] Sturlaugson AL, Fruchey KS, Fayer MD. Orientational dynamics of room temperature ionic liquid/water mixtures: Water-induced structure. J Phys Chem B 2012; 116: 177787.

http://dx.doi.org/10.1021/jp209942r

[35] Jiang W, Wang $Y$, Voth GA. Molecular dynamics simulation of nanostructural organization in ionic liquid/water mixtures. J Phys Chem B 2007; 111: 4812-8. http://dx.doi.org/10.1021/jp067142/

[36] Roth C, Appelhagen A, Jobst N, Ludwig R. Microheterogeneities in ionic-liquid-methanol solutions studied by FTIR Spectroscopy, DFT Calculations and molecular dynamics simulations. Chem Phys Chem 2012; 13: 1708-17.

http://dx.doi.org/10.1002/cphc.201101022

[37] Raabe G, Köhler J. Thermodynamical and structural properties of binary mixtures of imidazolium chloride ionic liquids and alcohols from molecular simulation. J Chem Phys 2012; 129: 144503-8.

http://dx.doi.org/10.1063/1.2990653 
[38] Maroncelli M, Fleming GR. Picosecond solvation dynamics of coumarin 153: The importance of molecular aspects of solvation. J Chem Phys 1987; 86: 6221-39. http://dx.doi.org/10.1063/1.452460

[39] Becke DA, Density-functional thermochemistry. III. The role of exact exchange. J Chem Phys 1993; 98: 5648-52. http://dx.doi.org/10.1063/1.464913

[40] Lee C, Yang W, Parr RG. Development of the Colic-Salvetti correlation-energy formula into a functional of the electron density. Phys Rev B 1988; 37: 785-9. http://dx.doi.org/10.1103/PhysRevB.37.785

[41] Frisch MJ, Trucks GW, Schlegel HB, Scuseria GE, Robb MA, Cheeseman JR, Zakrzewski VG, Montgomery JA, Stratmann RE Jr, Burant JC, Dapprich S, Millam JM, Daniels AD, Kudin KN, Strain MC, Farkas O, Tomasi J, Barone V, Cossi M, Cammi R, Mennucci B, Pomelli C, Adamo C, Clifford S, Ochterski J, Petersson GA, Ayala PY, Cui Q, Morokuma K, Malick DK, Rabuck AD, Raghavachari K, Foresman JB, Cioslowski J, Ortiz JV, Stefanov BB, Liu G, Liashenko A,
Piskorz P, Komaromi I, Gomperts R, Martin RL, Fox DJ, Keith T, Al Laham MA, Peng Y, Nanayakkara CA, Gonzalez C, Challacombe M, Gill PMW, Johnson B, Chen W, Wong MW, Andres JL, Gonzalez C, Head- Gordon M, Replogle ES, Pople JA, Gaussian 03; RevisionC.02: Gaussian, Inc.: Wallingford, CT 2004.

[42] Rahim Z, Barman BN, Acta Crystallographica A 1978; 34 761-4. http://dx.doi.org/10.1107/S0567739478001576

[43] Horng ML, Gardecki J, Papazyan A, Maroncelli M. Subpicosecond measurements of polar solvation dynamics: Coumarin 153 revisited. J Phys Chem 1995; 99: 17311-37. http://dx.doi.org/10.1021/j100048a004

[44] Horng ML, Gardecki J, Maroncelli M. Rotational dynamics of Coumarin 153: Time-dependent friction, dielectric friction, and other nonhydrodynamic effects. J Phys Chem A 1997; 101: 1030-47.

http://dx.doi.org/10.1021/jp962921v 